\title{
Nonlinear impedance of a microwave-driven Josephson junction with noise
}

\author{
W. T. Coffey \\ Department of Electronic and Electrical Engineering, Trinity College, Dublin 2, Ireland \\ J. L. Déjardin and Yu. P. Kalmykov* \\ Centre d'Études Fondamentales, Université de Perpignan, 52 Avenue de Villeneuve, 66860 Perpignan Cedex, France
}

(Received 21 September 1999)

\begin{abstract}
The nonlinear impedance of a point Josephson junction is calculated under various conditions for the resistively shunted junction model in the presence of noise. The calculation proceeds by solving the Langevin equation for the mechanical problem of a Brownian particle in a tilted cosine potential in the presence of a strong ac force ignoring inertial effects. The exact solution of the infinite hierarchy of equations for the moments (expectation values of the Fourier components of the phase angle), which describe the dynamics of the junction, is expressed in terms of a matrix continued fraction. This solution allows one to evaluate the nonlinear response of the junction (nonlinear microwave impedance, for example) to an ac microwave current of arbitrary amplitude. Strong nonlinear effects in the resistance and the reactance are observed for large ac currents as is demonstrated by plotting the nonlinear response characteristics as a function of the model parameters. For weak ac currents and low noise strengths, our results agree closely with previously available linear response and nonlinear response noiseless solutions, respectively. Applications of the model to the interpretation of recent experimental data found in the literature for the nonlinear behavior of microwave impedance of superconducting weak links are discussed.
\end{abstract}

\section{INTRODUCTION}

For several decades there has been intense interest in the effect of noise on the static and dynamic characteristics of Josephson junctions. The theoretical description of the effects of thermal fluctuations in superconducting weak links has been developed by constructing and attempting to solve the Fokker-Planck equation for the distribution function of the phase by analogy with the problem of the Brownian motion of a particle in a tilted periodic potential. The model has been applied to both dc and ac Josephson effects and to the driven Josephson oscillator (see, for example, Refs. 1-7). The problem of the Brownian motion of a particle in a tilted periodic potential also arises in a number of other physical applications: quantum noise in the ring-laser gyroscope, ${ }^{8,9}$ mobility of superionic conductors, ${ }^{10}$ laser with injected signal, ${ }^{11}$ theory of phase-locking techniques in radio engineering, ${ }^{12}$ etc. A related problem is the longitudinal dielectric relaxation of an assembly of single axis rotators in a $\cos N \theta$ potential. ${ }^{13} \mathrm{~A}$ comprehensive discussion of this model is given in Refs. 14 and 15 while the common features of the Josephson junction ac response in the presence of noise and dielectric relaxation have been emphasized in Ref. 13.

We proceed by recalling that Josephson junction devices are very sensitive to microwave signals. ${ }^{16}$ For example, if a Josephson junction is driven by an external ac (microwave) field, characteristic features (such as alteration of the overall shape of the current-voltage characteristics with the microwave power and the appearance of Shapiro steps ${ }^{17}$ corresponding to phase locking of the Josephson oscillator to the ac field) are observed and have been explained, for example, in Refs. 5 and 18-21. The calculation of the response of Josephson devices to an ac signal is generally referred to as the junction impedance problem. ${ }^{22} \mathrm{~A}$ knowledge of the junc- tion impedance is required in matching the junction to the external high-frequency current so as to achieve maximum power transfer. The calculation of the impedance $Z(\omega)$ of a point Josephson junction ignoring thermal noise effects has been given in many papers (see, e.g., Refs. 16 and 23 and references cited therein). Accounts of noise effects based on a numerical solution of the Fokker-Planck equation have been given in Ref. 24 (see also the discussion of these results in Ref. 25). Another method of solution of this equation for a similar problem (a ring-laser gyroscope) has been suggested by Cresser et al $^{26}$ in terms of infinite continued fractions. Further development of this continued fraction approach for the calculation of $Z(\omega)$ has been given in Refs. 27 and 28. However, all the above solutions are valid only for a weak ac signal and so pertain to the linear response. The calculation of the nonlinear impedance of a Josephson junction requires the evaluation of the time-dependent nonlinear response to a strong ac current, which is a much more complicated problem than calculating, for example, the dc current-voltage characteristics, where it is sufficient to find only a time-independent stationary solution of the Langevin (or the corresponding Fokker-Planck) equation. The calculation of the time-dependent portion of the nonlinear ac response requires therefore the application of the nonlinearresponse theory, which has not yet been developed up to now (in contrast to well-documented linear-response theory). Attempts to calculate the nonlinear ac response (mainly, the microwave resistance and reactance) to the strong probing ac current have been made by many authors, mainly, by using the perturbation theory, which is valid for low ac current amplitudes only, or in the noiseless limit, where the underlying nonlinear equation of motion can be solved numerically (see, e.g., Refs. 23, 29 and 30 and references cited therein). To our knowledge, the nonlinear ac response has 


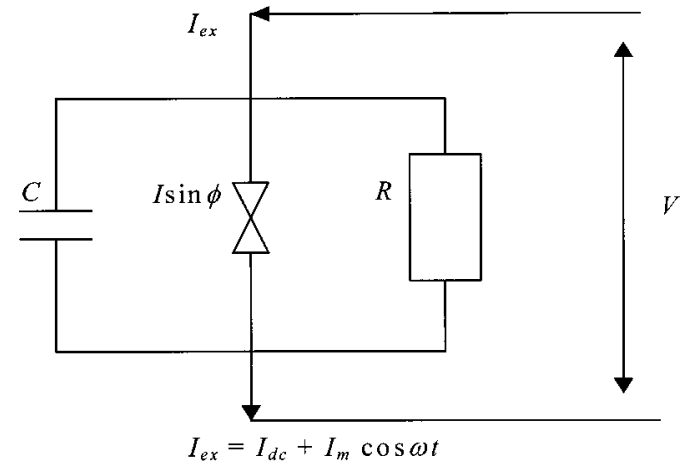

FIG. 1. Equivalent circuit of the Josephson junction.

not yet been analyzed systematically for large values of the ac current in the presence of noise. Here the nonlinear ac response of a point Josephson junction is evaluated in the presence of noise using the matrix continued fraction technique suggested in Ref. 31. This approach is, in some respects, equivalent to those used in Ref. 8 for the evaluation of the mean beat frequency of the dithered-ring-laser gyroscope and in Ref. 32 for the calculation of the harmonic mixing signal in a cosine potential. However, the solution obtained here differs from the solutions of Refs. 8 and 32 (principally because the time-dependent portion of the response may now be evaluated). Here the matrix continued fraction method allows one to obtain an exact solution of the infinite hierarchy of recurrence equations for the statistical averages governing the dynamics of the Josephson junction in the zero capacitance (noninertial) limit. Thus one may evaluate in the context of the resistively shunted junction (RSJ) model all the nonlinear response characteristics of the Josephson junction in the presence of noise and a strong ac current.

\section{RSJ MODEL}

The Josephson junction comprises two superconductors separated from each other by a thin layer of oxide. ${ }^{14}$ The phase difference $\phi=\phi_{l}-\phi_{r}$ between the wave functions of the right and left superconductors is given by the Josephson equation $^{14}$

$$
\frac{d}{d t} \phi(t)=\frac{2 e V(t)}{\hbar}
$$

where $V(t)$ is the potential difference across the oxide layer, $e$ is the charge of the electron, and $\hbar=h / 2 \pi$, where $h$ is the Planck constant. If the junction is small enough, i.e., the junction width is smaller than the Josephson penetration depth $\lambda_{J}$, it may be modeled ${ }^{14,15}$ (see Fig. 1) by a resistance $R$ in parallel with a capacitance $C$ across which is connected a current generator $I_{\mathrm{ex}}$ (representating the bias current applied to the junction). We suppose that the current $I_{\mathrm{ex}}$ consists of a dc current $I_{\mathrm{dc}}$ and an ac current $I_{\mathrm{ac}}=I_{m} \cos \omega t$. At the other end of the junction (across the resistance $R$ ) is connected a phase-dependent current generator, $I \sin \phi$, representing the Josephson supercurrent due to the Cooper pairs tunneling through the junction. Since the junction operates at a temperature above absolute zero, there will be a noise cur- rent $L(t)$ superimposed on the bias current. The currentbalance equation for the junction is then ${ }^{14}$

$$
\begin{aligned}
\frac{\hbar C}{2 e} & \frac{d^{2}}{d t^{2}} \phi(t)+\frac{\hbar}{2 e R} \frac{d}{d t} \phi(t)+I \sin \phi(t) \\
& =I_{\mathrm{dc}}+I_{m} \cos \omega t+L(t),
\end{aligned}
$$

where it is assumed that $L(t)$ is Gaussian white noise so that

$$
\overline{L(t)}=0, \overline{L(t) L\left(t^{\prime}\right)}=\frac{2 k T}{R} \delta\left(t-t^{\prime}\right) \text {. }
$$

Here $k$ is the Boltzmann constant, $T$ is the temperature, and the overbar means statistical averaging over an ensemble of junctions, which have all started at some instant $t$ with the same (sharp) values of $\phi$ and $\dot{\phi}$. We further simplify Eq. (2) by neglecting the effect of the capacitance $C$ on the dynamical behavior of the junction. This also restricts the range of frequencies in which the model is applicable, viz., $\omega \ll \omega_{p}$, where $\omega_{p}=\sqrt{2 I e / \hbar C}$ is the Josephson plasma frequency. This approximation can be justified if $C$ is small enough and has been discussed elsewhere. ${ }^{4}$ Equation (2) now becomes

$$
\tau_{J} \frac{d}{d t} \phi(t)-x-\xi \cos \omega t+\sin \phi(t)=I^{-1} L(t)
$$

where $x=I_{\mathrm{dc}} / I$ and $\xi=I_{m} / I$ are the ratios of the dc and ac current amplitudes to the supercurrent amplitude (tilt and nonlinear parameters $), \tau_{J}=\hbar /(2 e I R)$ is the characteristic relaxation time. Equation (3) is the noninertial Langevin equation for the RSJ model with noise.

\section{EVALUATION OF THE NONLINEAR RESPONSE IN TERMS OF MATRIX CONTINUED FRACTIONS}

Making the transformation $\phi \rightarrow e^{-i n \phi}$ in Eq. (3) and averaging the equation so obtained (as described in detail in Ref. 28) yields a system of differential-recurrence relations for the moments $\left\langle r^{n}\right\rangle=\left\langle e^{-i n \phi}\right\rangle$, viz.,

$$
\begin{aligned}
\tau_{J} \frac{d}{d t}\left\langle r^{n}\right\rangle(t)+\left[i n(x+\xi \cos \omega t)+n^{2} / \gamma\right]\left\langle r^{n}\right\rangle(t) \\
\quad=\frac{n}{2}\left[\left\langle r^{n-1}\right\rangle(t)-\left\langle r^{n+1}\right\rangle(t)\right],
\end{aligned}
$$

where $\gamma=\hbar I /(2 e k T)$ is the noise strength parameter $(\gamma$ $\rightarrow \infty$ corresponds to the noiseless limit) and the angular brackets mean statistical averaging over the sharp values of $\phi$ at time $t$. Here $\gamma$ differs by a factor $\frac{1}{2}$ from that used in Refs. 27 and 28. In order to obtain Eq. (4) we have used the Stratonovich definition of a stochastic differential equation ${ }^{33}$ as that definition is the mathematical idealization of the noninertial relaxation process considered here. Thus it is unnecessary to transform the Langevin Eq. (3) to an Itô equation (e.g., Ref. 15) and so the methods of ordinary analysis apply.

The quantities of physical interest are the mean value of the voltage $\langle V\rangle$ and the junction impedance to an external high-frequency current. One may evaluate these quantities from the following equation: 


$$
\frac{\langle V\rangle}{I R}=x+\xi \cos \omega t-\langle\sin \phi\rangle(t),
$$

where

$$
\langle\sin \phi\rangle(t)=\frac{i}{2}\left[\langle r\rangle(t)-\left\langle r^{-1}\right\rangle(t)\right] .
$$

In order to accomplish this, we note that the stochastic dynamics of the junction in the presence of the ac current $I(t)=I_{m} \cos \omega t$, which is described by the noninertial Langevin Eq. (3), is a nonstationary Markovian process. Here we are solely concerned with the stationary ac response, which is independent of the initial condition, so that one needs to calculate the solution of Eq. (3) corresponding to the stationary state. To accomplish this, one may seek all the $\left\langle r^{n}\right\rangle(t)$ in the form

$$
\left\langle r^{n}\right\rangle(t)=\sum_{k=-\infty}^{\infty} F_{k}^{n}(\omega) e^{i k \omega t} .
$$

On substituting Eq. (7) into Eq. (4), we have the following recurrence equations for the Fourier amplitudes $F_{k}^{n}(\omega)$, namely,

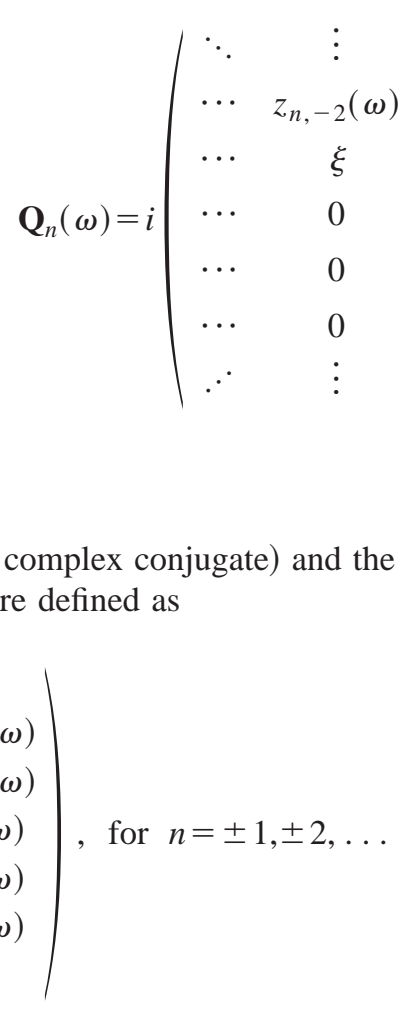

and

$$
\mathbf{C}_{0}(\omega)=\mathbf{C}_{0}=\left(\begin{array}{c}
\vdots \\
0 \\
0 \\
1 \\
0 \\
0 \\
\vdots
\end{array}\right), \text { for } n=0
$$

$$
\begin{aligned}
& F_{k}^{n+1}(\omega)+i z_{n, k}(\omega) F_{k}^{n}(\omega)+i \xi\left[F_{k-1}^{n}(\omega)+F_{k+1}^{n}(\omega)\right] \\
& \quad-F_{k}^{n-1}(\omega)=0
\end{aligned}
$$

where $n$ and $k$ vary from $-\infty$ to $\infty$, and

$$
z_{n, k}(\omega)=2\left(x+\omega \tau_{J} k / n-i n / \gamma\right)
$$

The scalar five-term recurrence Eq. (8) can be transformed into the two uncoupled matrix three-term recurrence relations

$$
\mathbf{Q}_{n}(\omega) \mathbf{C}_{n}(\omega)+\mathbf{C}_{n+1}(\omega)=\mathbf{C}_{n+1}(\omega) \quad(n=1,2,3, \ldots)
$$

and

$$
\begin{array}{r}
-\mathbf{Q}_{n}^{*}(-\omega) \mathbf{C}_{-n}(\omega)+\mathbf{C}_{-n-1}(\omega) \\
\quad=\mathbf{C}_{-n+1}(\omega) \quad(n=1,2,3, \ldots),
\end{array}
$$

where $\mathbf{Q}_{n}$ is a tridiagonal infinite matrix given by

Thus, in order to calculate $\langle\sin \phi\rangle(t)$ in Eq. (5), we need to evaluate $\mathbf{C}_{1}(\omega)$ and $\mathbf{C}_{-1}(\omega)$, which contain all the Fourier amplitudes of $\langle r\rangle(t)$ and $\left\langle r^{-1}\right\rangle(t)$. Equation (10) can be solved for $\mathbf{C}_{1}$ in terms of matrix continued fractions, ${ }^{15,28}$ viz.,

$$
\mathbf{C}_{1}(\omega)=\frac{\mathbf{I}}{\mathbf{Q}_{1}(\omega)+\frac{\mathbf{I}}{\mathbf{Q}_{2}(\omega)+\frac{\mathbf{I}}{\mathbf{Q}_{3}(\omega)+\cdots}}} \mathbf{C}_{0},
$$

where the fraction lines designate the matrix inversions and I is the identity matrix of infinite dimension. Having determined $\mathbf{C}_{1}(\omega)$, it is not necessary to solve Eq. (11) for $\mathbf{C}_{-1}(\omega)$, as all the components of the column vector $\mathbf{C}_{-1}(\omega)$ can be obtained from Eq. (14), on noting that

$$
F_{0}^{-1}(\omega)=F_{0}^{1^{*}}(\omega) \text { and } F_{k}^{-1}(\omega)=-F_{-k}^{1^{*}}(\omega) \quad(\text { for } k \neq 0) \text {. }
$$

On using Eqs. (14) and (15), one can now calculate from Eqs. (5) and (6) both the time independent (but frequency dependent) dc $I-V$ characteristic in the presence of an ac current 


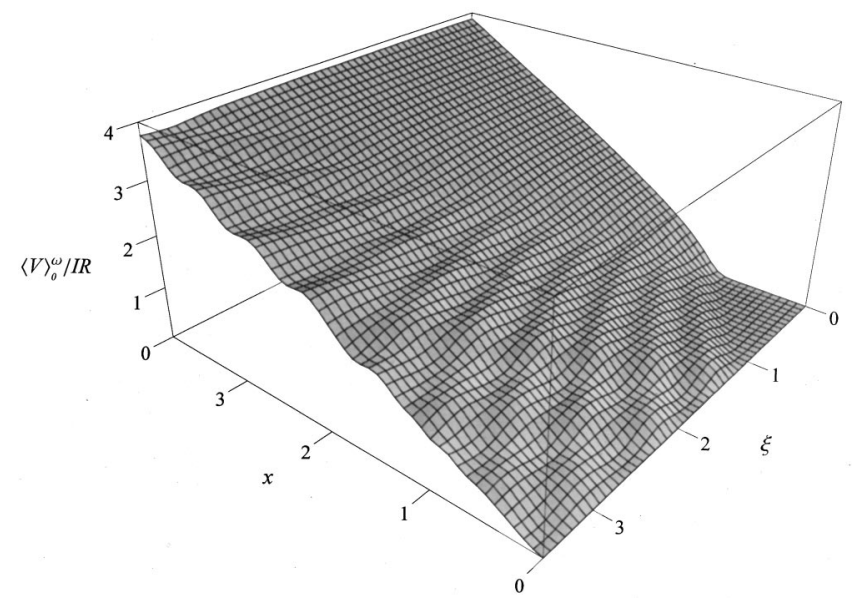

FIG. 2. $\langle V\rangle_{0}^{\omega} / I R$ vs $\xi$ and $x\left(\gamma=25, \omega \tau_{J}=0.3\right)$, showing the Shapiro steps, which become pronounced at high $x$ values as the amplitude $\xi$ of the ac current increases. In the limit $\xi \rightarrow 0$, the dc current characteristic is given by Eq. (23).

$$
\langle V\rangle_{0}^{\omega} / I R=x+\operatorname{Im}\left\{F_{0}^{1}(\omega)\right\}
$$

and the stationary ac response

$$
\frac{\left\langle V-\langle V\rangle_{0}^{\omega}\right\rangle}{I R}=\sum_{k=1}^{\infty} \xi^{k} \operatorname{Re}\left\{Z_{k}(\omega) e^{i k \omega t}\right\},
$$

where

$$
Z_{k}(\omega)=\delta_{1, k}-\frac{i}{\xi^{k}}\left[F_{k}^{1}(\omega)+F_{-k}^{1^{*}}(\omega)\right]
$$

and $\delta_{i, k}$ is Kronecker's delta. Equation (18) for $k=1$ yields the nonlinear impedance $Z(\omega)$, viz.,

$$
Z(\omega)=R_{\omega}-i X_{\omega}=R Z_{1}(\omega),
$$

where $R_{\omega}$ and $X_{\omega}$ are the dynamic resistance and the reactance, respectively. The limiting case of a weak ac current allows one to calculate the linear impedance (i.e., the impedance in the linear response approximation). Indeed, if the ac current $I_{m} e^{i \omega t}$ is weak, so that $\hbar I_{m} / 2 e k T \ll 1$, one has from Eqs. (5) and (17)

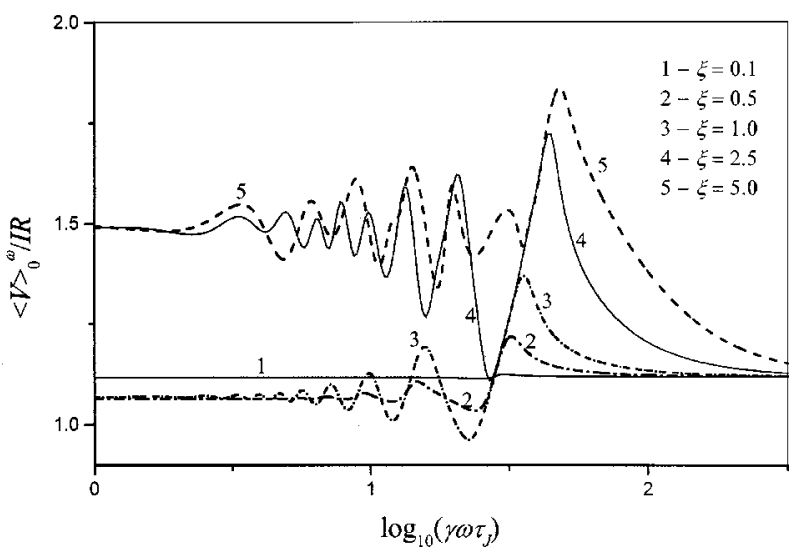

FIG. 3. $\langle V\rangle_{0}^{\omega} / I R$ vs $\log _{10}\left(\gamma \omega \tau_{J}\right)$ for various $\xi(\gamma=25, x=1.5)$. Strong nonlinearity corresponding to large $\xi$ causes pronounced oscillatory behavior of the voltage with numerous troughs and peaks.

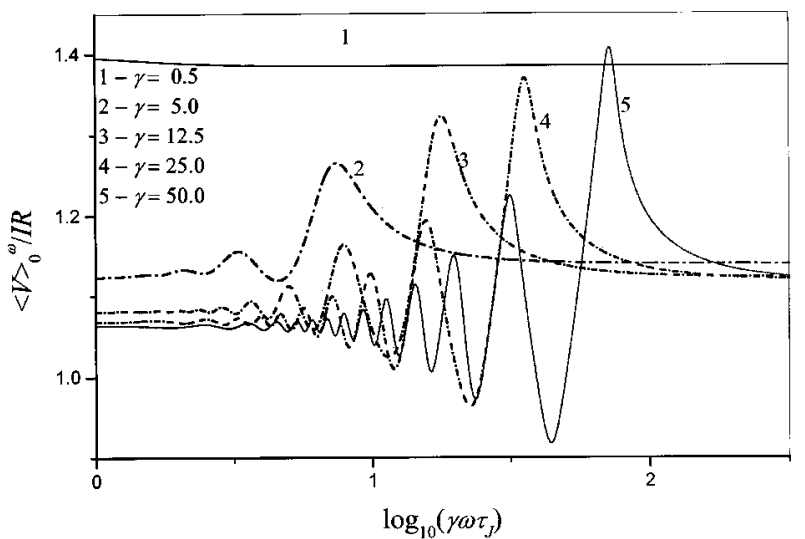

FIG. 4. $\langle V\rangle_{0}^{\omega} / I R$ vs $\log _{10}\left(\gamma \omega \tau_{J}\right)$ for various $\gamma(\xi=1$ and $x$ $=1.5$ ). The effect of decreasing $\gamma$ is to greatly decrease the oscillatory behavior so that the characteristic becomes frequency independent.

$$
\langle V\rangle=\langle V\rangle_{0}+\langle V\rangle_{1},
$$

where

$$
\langle V\rangle_{0} / I R=x-\langle\sin \phi\rangle_{0}
$$

and

$$
\langle V\rangle_{1}=Z(\omega) I_{m} e^{i \omega t} .
$$

Here the subscripts " 0 " and " 1 " in the angular braces denote the average in the absence of the ac current and the portion of the average which is linear in $I_{m}$, respectively. Thus Eq. (21) describes the dc $I-V$ characteristic of the junction, while Eq. (22) yields its linear impedance.

\section{RESULTS AND DISCUSSION}

The exact matrix continued fraction solution [Eq. (14)] just obtained is very convenient for the purpose of computation (algorithms for calculating matrix continued fractions are discussed in Ref. 15, Chap. 9). This matrix continued fraction satisfies the conditions of a theorem (proved in Ref. 34) appropriate to the convergence of matrix continued fractions. This theorem guarantees the convergence of a matrix

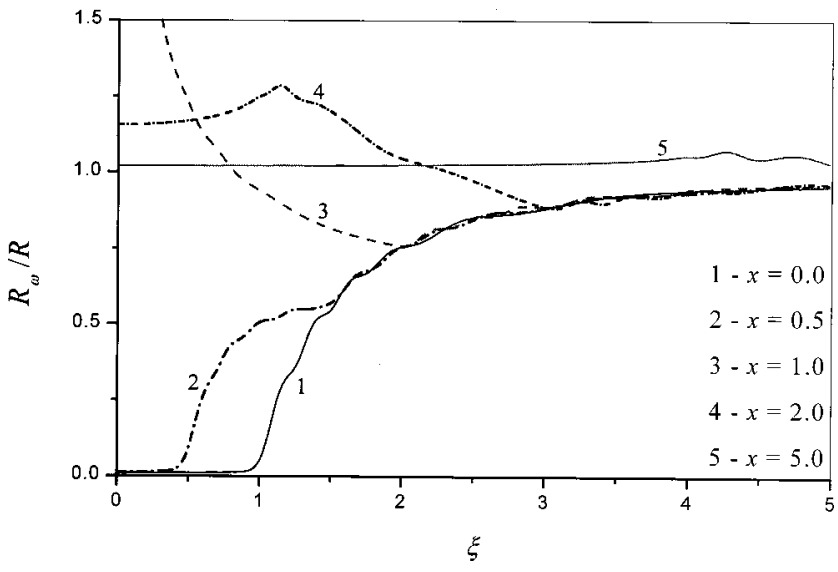

FIG. 5. Resistance $R_{\omega}$ vs $\xi$ for various dc bias current amplitudes $x\left(\gamma=50, \omega \tau_{J}=0.1\right)$, showing strong nonlinearity for small bias and Ohmic resistancelike behavior for high bias. 


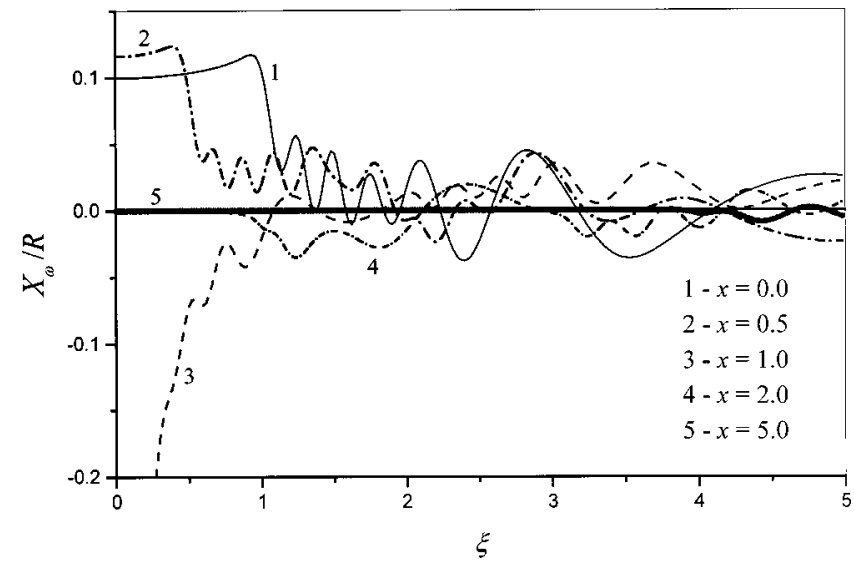

FIG. 6. The same as in Fig. 5 for the reactance $X_{\omega}$.

continued fraction if it satisfies two definite conditions, which for the present problem are

$$
4 \operatorname{Det}\left(\mathbf{Q}_{n}^{-1}\right) \operatorname{Det}\left(\mathbf{Q}_{n+1}^{-1}\right) \leqslant 1 \quad(\text { for all } n)
$$

and

$$
\lim _{n \rightarrow \infty} 4^{n} \prod_{k=0}^{n} \operatorname{Det}\left(\mathbf{Q}_{k+2}^{-1}\right) \operatorname{Det}\left(\mathbf{Q}_{k+1}^{-1}\right)=0
$$

As the matrix $\mathbf{Q}_{n}$ in Eq. (12) fulfils the above conditions, the matrix continued fraction in Eq. (14) converges. In the calculation, the infinite matrix continued fraction in Eq. (14) was approximated by a matrix continued fraction of finite order (by putting $\mathbf{Q}_{n}=\mathbf{0}$ at some $n=N$ ) and the dimension of the matrix $\mathbf{Q}_{n}$ and the vectors $\mathbf{C}_{0}$ was confined to some finite number $M$. Both $N$ and $M$ depend on the parameters $\gamma$ and $\xi$ and on the number of harmonics to be taken into account. They must be chosen by stipulating the accuracy of the calculation a priori. For example, in the calculation of the $F_{k}^{1}(\omega)$ for $k$ up to 8 and for $\xi$ up to 10 , the dimensions of $\mathbf{Q}_{n}$ and $\mathbf{C}_{0}$ need not exceed 50 and 15-25 iterations in calculating the matrix continued fraction are enough to arrive at an accuracy of not less than six significant digits in the majority of cases.

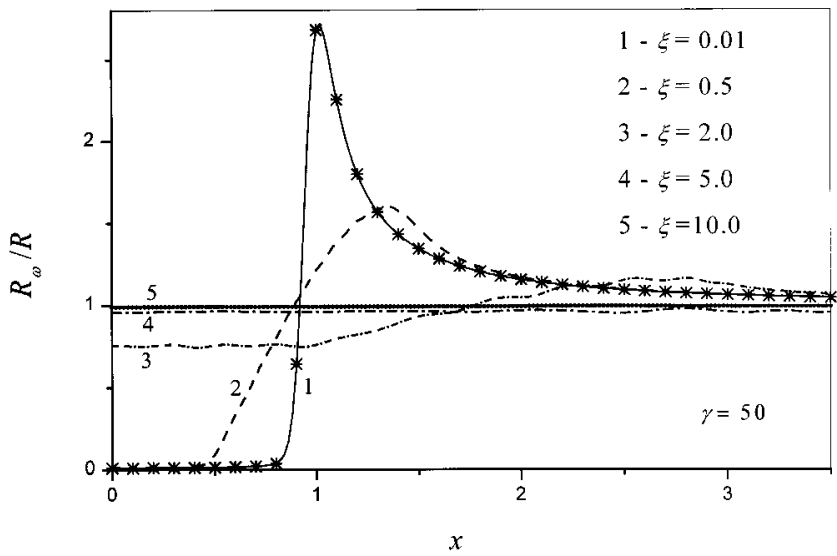

FIG. 7. Resistance $R_{\omega}$ vs $x$ for various $\xi\left(\gamma=50, \omega \tau_{J}=0.1\right)$, showing pronounced departure from linear response (curve 1) as ac current amplitude is increased. Stars correspond Eq. (24).

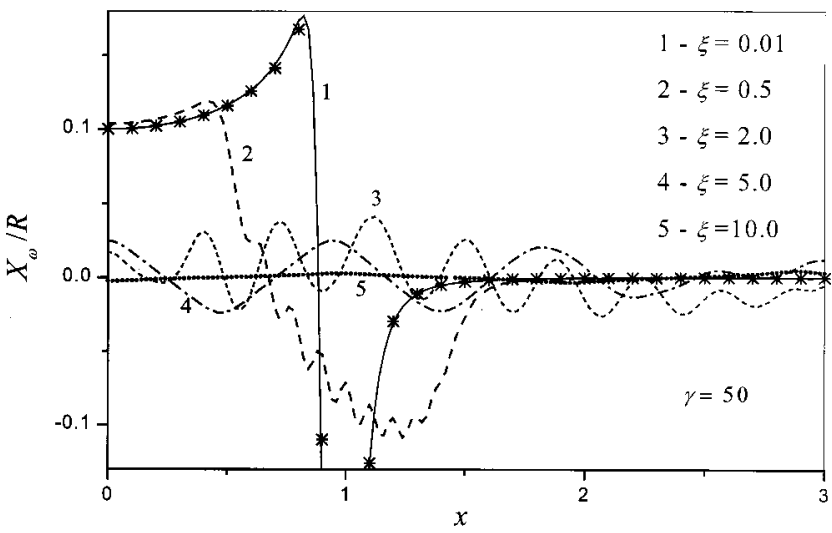

FIG. 8. The same as in Fig. 7 for $X_{\omega}$.

For $\xi=0$ Eqs. (14) and (16) yield the dc $I-V$ characteristic, which is in complete agreement with the results of Ambegaokar and Halperin ${ }^{1}$ who computed this characteristic by solving the noninertial (when the capacitance is neglected) Fokker-Planck equation associated with the Langevin Eq. (3). Their results may be presented as follows: ${ }^{28}$

$$
\frac{\langle V\rangle_{0}}{I R}=x+\operatorname{Im}\left\{\frac{I_{1+i x \gamma}(\gamma)}{I_{i x \gamma}(\gamma)}\right\},
$$

where $I_{v}(z)$ is the modified Bessel function of the first kind of order $v{ }^{35}$ There are three simple approximations of Eq. (23) for extreme cases, ${ }^{1}$ i.e., (i) $\gamma \rightarrow 0:\langle V\rangle_{0}=I_{\mathrm{dc}} R$-this limit corresponds to Ohm's law, (ii) $\gamma \rightarrow \infty:\langle V\rangle_{0}=0(x$ $<1)$ - pure superconduction, and (iii) $\langle V\rangle_{0}=\operatorname{IR}\left(x^{2}-1\right)^{1 / 2}$ $(x>1)$-which is Ohm's law with a correction factor. In the presence of a strong ac current the $I-V$ characteristics behave in a very interesting way. For low noise strengths (say $\gamma$ $=25$ ), the shape of the $I-V$ characteristics shown in Fig. 2 become distorted when $\xi$ increases and the Shapiro steps induced on the curves adhere to the Ohmic line $\langle V\rangle_{0}$ $=I_{\mathrm{dc}} R$. The frequency spectra of $\langle V\rangle_{0}^{\omega} / I R$ (Figs. 3 and 4 ) show how the oscillatory behavior appears with increasing $\xi$ and $\gamma$. The nonlinear features shown in Figs. 2-4 disappear for low driving frequencies $\left(\omega \tau_{j} \leqslant \gamma^{-1}\right)$ and/or high noise strengths $(\gamma \leqslant 1)$.

Strong nonlinear effects also appear in the ac impedance. In Figs. 5 and 6, the resistance $R_{\omega}$ and the reactance $X_{\omega}$ of

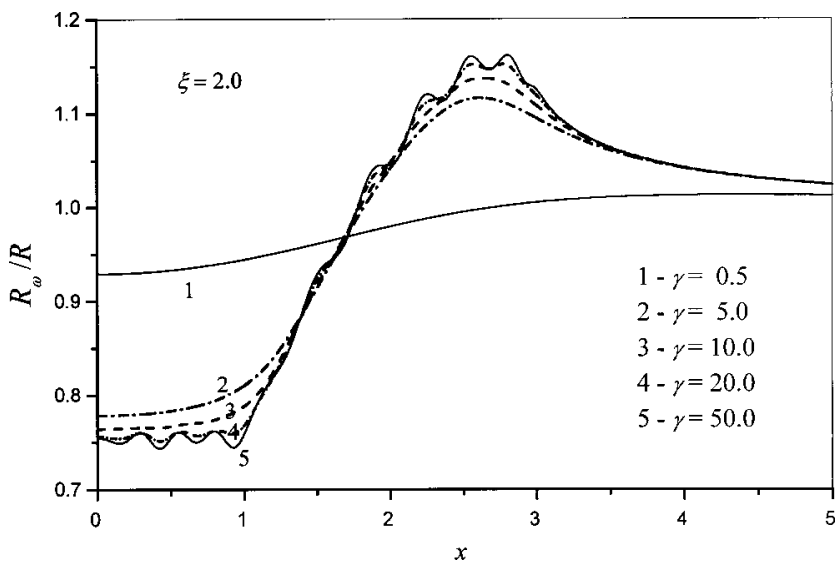

FIG. 9. Resistance $R_{\omega}$ vs $x$ for various $\gamma$ at $\xi=2.0$ and $\omega \tau_{J}$ $=0.1$, showing pronounced nonlinear effects for large $\gamma$. 


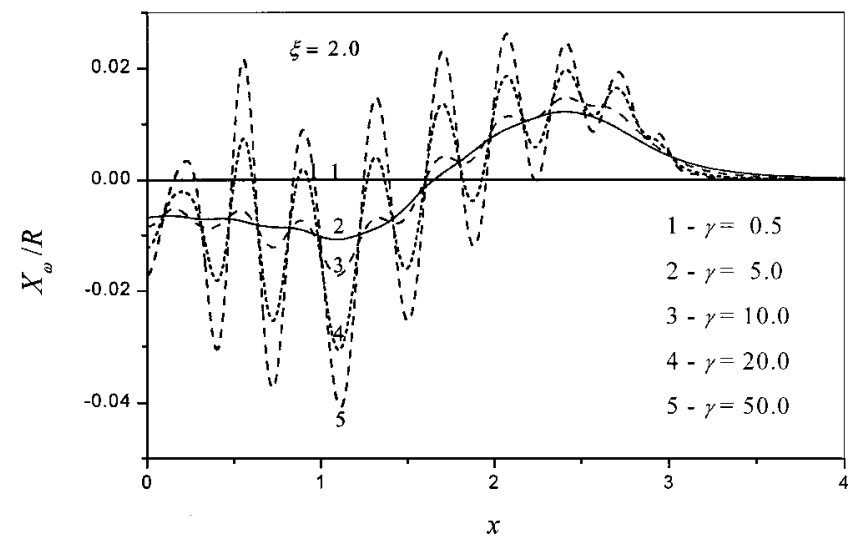

FIG. 10. The same as in Fig. 9 for $X_{\omega}$.

the junction are plotted versus the nonlinear parameter $\xi$. For comparison we have chosen the value of the parameter $\omega \tau_{J}$ $=0.1$, as was used in Ref. 30 for the calculation of the nonlinear impedance in the noiseless limits. Just as in that limit, in the presence of noise, the behavior of $R_{\omega}$ vs $\xi$ at small dc bias exhibits both a threshold field and a parabolic increase above the threshold with associated steplike structures. When $I_{\mathrm{dc}}$ is larger than the dc critical current $(x>1), R_{\omega}$ suddenly becomes greater than $R$. For large values of the dc bias, the influence of the ac current diminishes and $R_{\omega}$ and $X_{\omega}$ approach the shunt resistance $R$ and zero, respectively. Figure 5 shows clearly that the resistive response saturates at large $\xi$. This result has also been obtained in the noiseless limit. ${ }^{29}$ The oscillatory behavior of the reactance $X_{\omega}$ vs $\xi$ is a manifestation of the dynamic phase slips, which occur as the ac current is increased (see Fig. 6). ${ }^{30}$ Strong nonlinear effects in both $R_{\omega}$ and $X_{\omega}$ versus the bias parameter $x$ for various $\xi$ and $\gamma$ are shown in Figs. 7-10. Figures 7 and 8 also emphasize the pronounced departure from linear response as $\xi$ is increased. For $\gamma \gg 1$, the above results are in quantitative agreement with those obtained in Ref. 30 in the noiseless limit. Furthermore, just as in the $I-V$ characteristic, the nonlinear features disappear at $\omega \tau_{J} \leqslant \gamma^{-1}$ and/or $\gamma \leqslant 1$. In Fig. 11 , we have also presented $\log _{10}\left(R_{\omega} / R\right)$ as a function of $\log _{10}\left(I_{m} / I_{0}\right)$ for various values of the normalized supercurrent $I / I_{0}$ at $x=0$ and $\omega \tau_{J}=0.1$. This figure clearly demon-

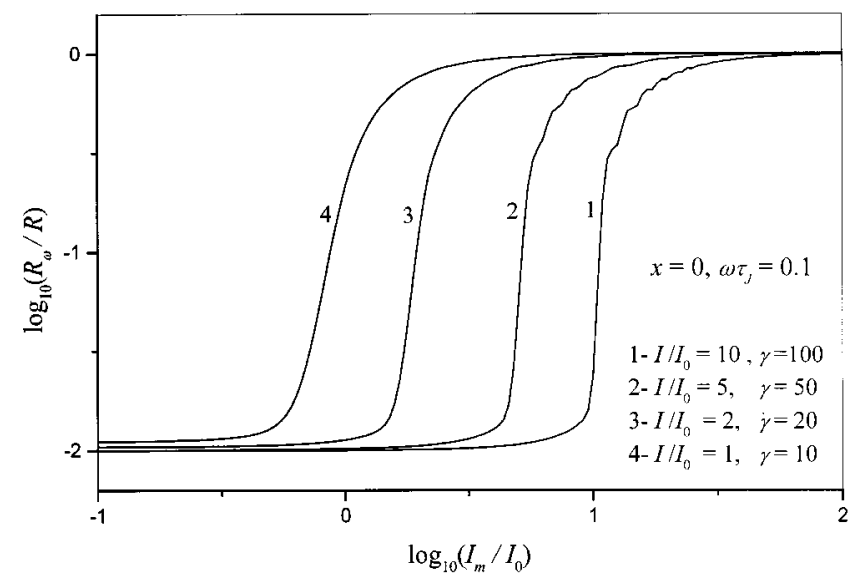

FIG. 11. $\log _{10}\left(R_{\omega} / R\right)$ vs $\log _{10}\left(I_{m} / I_{0}\right)$ for various values of $I / I_{0}$ and $\gamma$ (such that $I /\left(I_{0} \gamma\right)=$ const) at $x=0$ and $\omega \tau_{J}=0.1$, showing pronounced nonlinear effects (threshold points and saturation).

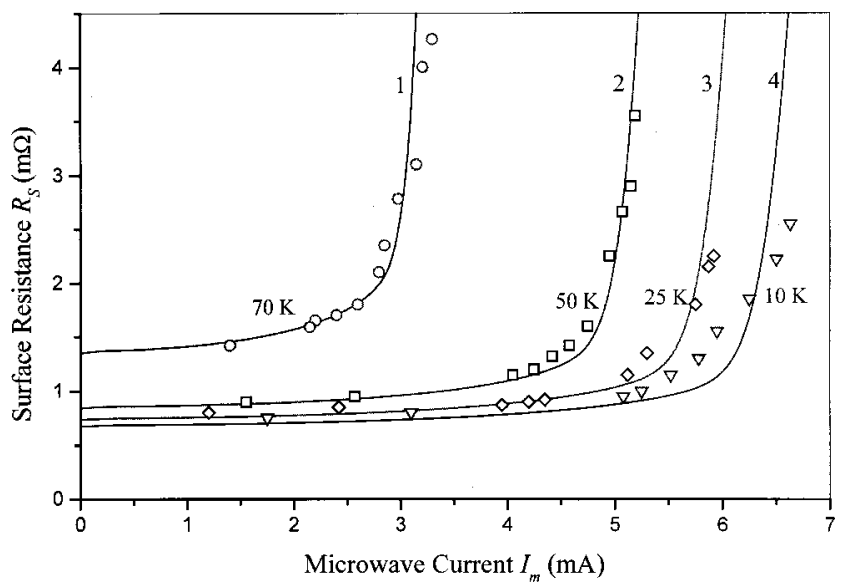

FIG. 12. Surface resistance $R_{s}$ dependence on microwave current in weak-link Josephson junctions in high- $T_{c}$ superconductors for different temperatures. Solid lines represent fits obtained from Eqs. (14), (15), and (19) for $\gamma=100$ (noiseless limit) and $\omega \tau_{J}$ $\approx 0.14$ (corresponding to the experimental frequency $14 \mathrm{GHz}$ ). Experiment: $70 \mathrm{~K}$ (circles), $50 \mathrm{~K}$ (squares), $25 \mathrm{~K}$ (diamonds), and 10 $\mathrm{K}$ (triangles) from Ref. 41.

strates pronounced nonlinear effects, viz., the steps at large $\gamma$ and $\xi$ (curve 1), the threshold points and saturation $\left(R_{\omega} \rightarrow R\right.$ at $\left.\xi \rightarrow \infty\right)$ of the nonlinear response.

For $\xi \ll 1$, the present theory is in accordance with the results of Refs. 27 and 28, where an approximate analytical equation for the linear impedance $Z(\omega)$ of the junction has been derived, viz.,

$$
\frac{Z(\omega)}{R}=1-\frac{1}{2}\left[\frac{I_{1+i x \gamma}(\gamma) / I_{i x \gamma}(\gamma)}{\lambda-i \omega \tau_{J}}+\frac{I_{1-i x \gamma}(\gamma) / I_{-i x \gamma}(\gamma)}{\lambda^{*}-i \omega \tau_{J}}\right]
$$

where $\lambda$ is given by

$$
\lambda=\frac{I_{i x \gamma}(\gamma) I_{1+i x \gamma}(\gamma)}{2 \int_{0}^{\gamma} I_{i x \gamma}(t) I_{1+i x \gamma}(t) d t} .
$$

For weak ac currents, Eq. (24) yields good correspondence to the exact solution Eq. (14) for wide ranges of $\omega, x$, and $\gamma$ (see, for example, Figs. 7 and 8).

The results obtained may be useful for the interpretation of experimental data for the microwave resistance and reactance of weak links. For example, experimental observations of nonlinear effects in the microwave resistance of a high quality single crystal $\mathrm{YBa}_{2} \mathrm{Cu}_{3} \mathrm{O}_{7-\delta}$, were presented and explained in Refs. 30 and 36 using the RSJ model. Experimental data ${ }^{37}$ on the nonlinear microwave surface impedance $Z_{s}$ of high-temperature superconducting thin films were also interpreted ${ }^{38-40}$ in the context of the RSJ model and a qualitative agreement of the model with experimental measurements of $Z_{s}$ was observed. It was shown there that the above systems behave like a Josephson junction and the RSJ model describes the main features of the nonlinear surface impedance: the steps in the ac current dependence of the resistance, ${ }^{30,36}$ the threshold points ${ }^{39,40}$ and saturation ${ }^{39,40}$ (see Fig. 11). Other experiments, which can be analyzed in the context of the RSJ model, are microwave absorption measurements in weak-link Josephson junctions ${ }^{41}$ in high- $T_{c}$ temperature superconductors, where the microwave surface 
resistance $R_{s}$ can also be studied. As was shown in Ref. 41, the numerical solution of the underlying nonlinear dynamic equation for the noiseless RSJ model [Eq. (3) with $L(t)=0$ ] agrees closely with experiment. For the purpose of illustration, a comparison of the theoretical results presented in this paper with experimental data ${ }^{41}$ on measurements of the microwave surface resistance $R_{s}$ of a Y-Ba-Cu-O film at 14 $\mathrm{GHz}$ for $70,50,25$, and $10 \mathrm{~K}$ is shown in Fig. 12. Here the solid lines represent the fits obtained from Eqs. (14), (15), (18), and (19) for $R_{\omega}$ in the low noise case $\gamma=100$, say (a further increase of $\gamma$ does not change the results). Each of the four temperature data was fitted with the same value of $I R$ $=212 \mu \mathrm{V}$ (as in Ref. 41), the following values of $I$ being obtained from the fits: $3.0 \mathrm{~mA}$ for $70 \mathrm{~K}, 4.8 \mathrm{~mA}$ for $50 \mathrm{~K}$, $5.5 \mathrm{~mA}$ for $25 \mathrm{~K}$, and $6.0 \mathrm{~mA}$ for $10 \mathrm{~K}$ (these values of $I$ are very close to those given in Ref. 41). As can be seen in Fig. 12 , the fitting is good and is very similar to that obtained in Ref. 41. This similarity is not surprising because the experimental conditions ${ }^{41}$ correspond to values of $\gamma \gg 1$ for which the RSJ model with noise agrees in all respects with that used in Ref. 41. The above estimates, as well as those given in Refs. 30 and 36-41, correspond to the noiseless case, $\gamma$ $\gg 1$. However, the theory presented here can also be applied to the analysis of the nonlinear impedance taking account of noise effects, which may provide a better quantitative agreement with experiments.
Thus in the present paper we have established formulas for the ac nonlinear response of a Josephson junction in the presence of noise for wide ranges of the nonlinearity $(\xi)$, the noise strength $(\gamma)$, and the bias $(x)$ model parameters. In order to obtain these results, we have used the matrix continued fraction approach, which allowed us to solve exactly the problem of the noninertial Brownian motion in a tilted cosine potential in the presence of a large ac force. Pronounced nonlinear effects appear in the response of the junction for $\gamma \gg 1$ and frequencies $\omega$ satisfying the condition $\gamma^{-1} \leqslant \omega \tau_{J} \leqslant 1$. As we have mentioned in the Introduction, a Langevin equation of the kind used in the present paper also arises in a number of other stochastic systems with a cosine potential in the overdamped limit ${ }^{15}$ subjected to a strong ac driving force. Therefore the results obtained here for the Josephson junction may be applied to other nonlinear systems (such as the dithered-ring-laser gyroscope $\mathrm{e}^{9}$ ), where the effect of noise is of importance.

\section{ACKNOWLEDGMENTS}

The support of this work by the International Association for the Promotion of Cooperation with Scientists from the New Independent States of the Former Soviet Union (Grant No. INTAS 96-0663) and by the French Ministry of Foreign Affairs (French Embassy in Ireland) is gratefully acknowledged.
*Author to whom correspondence should be addressed.

${ }^{1}$ V. Ambegaokar and B. I. Halperin, Phys. Rev. Lett. 22, 1364 (1969).

${ }^{2}$ Yu. M. Ivanchenko and L. A. Zil'berman, Zh. Eksp. Teor. Phys. 55, 2395 (1968) [ Sov. Phys. JETP 28, 1272 (1969)].

${ }^{3}$ M. P. Stephen, Phys. Rev. Lett. 21, 1629 (1968).

${ }^{4}$ M. P. Stephen, Phys. Rev. 186, 393 (1969).

${ }^{5}$ W. H. Henkels and W. W. Webb, Phys. Rev. Lett. 26, 164 (1971).

${ }^{6}$ P. A. Lee, J. Appl. Phys. 42, 325 (1971).

${ }^{7}$ V. Berdichevsky and M. Gitterman, Phys. Rev. E 56, 6340 (1997).

${ }^{8}$ W. Schleich, C.-S. Cha, and J. D. Cresser, Phys. Rev. A 29, 230 (1984).

${ }^{9}$ W. W. Chow, J. Gea-Banacloche, L. M. Pedrotti, V. Sanders, W. Schleich, and M. O. Scully, Rev. Mod. Phys. 57, 61 (1985).

${ }^{10}$ W. Dieterich, P. Fulde, and I. Peschel, Adv. Phys. 29, 527 (1980).

${ }^{11}$ W. W. Chow, M. O. Scully, and E. W. Van Stryland, Opt. Commun. 15, 6 (1975).

${ }^{12}$ A. J. Viterbi, Principles of Coherent Communication (McGrawHill, New York, 1966).

${ }^{13}$ W. T. Coffey, Adv. Chem. Phys. 63, 69 (1985).

${ }^{14}$ G. Barone and A. Paterno, Physics and Applications of the Josephson Effect (Wiley, New York, 1982).

${ }^{15}$ H. Risken, The Fokker-Planck Equation, 2nd ed. (SpringerVerlag, Berlin, 1989).

${ }^{16}$ F. Auracher and T. Van Duzer, J. Appl. Phys. 44, 848 (1973).

${ }^{17}$ S. Shapiro, Phys. Rev. Lett. 11, 80 (1963).

${ }^{18}$ C. Vanneste, C. C. Chi, K. H. Brown, A. C. Callegari, M. M. Chen, J. H. Greiner, H. C. Jones, K. K. Kim, A. W. Kleinsasser, H. A. Notarys, G. Proto, R. H. Wang, and T. Yogi, Phys. Rev. B 31, 4230 (1985)

${ }^{19}$ W. C. Danchi, J. B. Hansen, M. Octavio, F. Habbal, and M. Tinknam, Phys. Rev. B 30, 2503 (1984).
${ }^{20}$ Q. Hu, C. J. Lobb, and M. Tinknam, Phys. Rev. B 35, 1687 (1987).

${ }^{21}$ E. Ben-Jacob and D. J. Bergman, Phys. Rev. E 29, 2021 (1984).

${ }^{22}$ C. V. Stancampiano, IEEE Trans. Electron Devices 27, 1934 (1980).

${ }^{23}$ H. Kanter and F. L. Vernon, Jr., J. Appl. Phys. 43, 3174 (1993).

${ }^{24}$ K. K. Likharev and V. K. Semenov, Radiotekh. Elektron. (Moscow) 18, 1767 (1973).

${ }^{25}$ A. N. Vystavkin, V. N. Gubankov, L. S. Kuzmin, K. K. Likharev, V. V. Migulin, and V. K. Semenov, Rev. Phys. Appl. 9, 79 (1974).

${ }^{26}$ J. D. Cresser, D. Hammonds, W. H. Louisell, P. Meystre, and H. Risken, Phys. Rev. A 25, 2226 (1982)

${ }^{27}$ W. T. Coffey, Yu. P. Kalmykov, and E. S. Massawe, Phys. Rev. E 48, 77 (1993).

${ }^{28}$ W. T. Coffey, Yu. P. Kalmykov, and J. T. Waldron, The Langevin Equation (World Scientific, Singapore, 1996), Chap. 6.

${ }^{29}$ J. McDonald and J. R. Clem, Phys. Rev. B 56, 14723 (1997).

${ }^{30}$ Z. Zhai, P. V. Parimi, and S. Sridhar, Phys. Rev. B 59, 9573 (1999).

${ }^{31}$ J. L. Déjardin and Yu. P. Kalmykov, Phys. Rev. E 61, 1211 (2000).

${ }^{32}$ H.-J. Breymayer, H. Risken, H. D. Vollmer, and W. Wonneberger, Appl. Phys. B: Photophys. Laser Chem. 28, 335 (1982).

${ }^{33}$ R. L. Stratonovich, Conditional Markov Processes and Their Application to the Theory of Optimal Control (Elsevier, New York, 1968).

${ }^{34}$ H. Denk and M. Riederle, J. Approx. Theory 35, 355 (1982).

${ }^{35} \mathrm{G}$. N. Watson, Theory of Bessel Functions, 2nd ed. (Cambridge University Press, Cambridge, 1944), p. 46.

${ }^{36}$ Z. Zhai, H. Srikanth, S. Sridhar, A. Erb, E. Walker, and R. 
Flukinger, Physica C 282-287, 1601 (1997).

${ }^{37}$ P. P. Nguen, D. E. Oats, G. Dresselhaus, and M. S. Dresselhaus, Phys. Rev. B 48, 6400 (1993).

${ }^{38}$ B. B. Jin and R. X. Wu, J. Appl. Phys. 84, 3250 (1998).

${ }^{39}$ Y. M. Habib, C. J. Lehner, D. E. Oats, L. R. Vale, R. H. Ono, G. Dresselhaus, and M. S. Dresselhaus, Phys. Rev. B 57, 13833
(1998).

${ }^{40}$ D. E. Oats, P. P. Nguyen, Y. M. Habib, G. Dresselhaus, M. S. Dresselhaus, G. Koren, and E. Polturak, Appl. Phys. Lett. 68, 705 (1996).

${ }^{41}$ L. M. Xie, J. Wosik, and J. C. Wolfe, Phys. Rev. B 54, 15494 (1996). 